\title{
DESENVOLVIMENTO DE UMA BANCADA DIDÁTICA PARA ANÁLISE COMPARATIVA DO DESEMPENHO DE VENTILADORES INDUSTRIAIS
}

DOI: 10.37702/2175-957X.COBENGE.2021.3426

Luiz Carlos Cordeiro Jr - luiz.cordeiro@fat.uerj.br

UERJ

Rua Benedito Alves de Moraes 157

27523-116 - Resende - RJ

Patrick Vinicius de Souza Teodoro - patrick.vinicius96@gmail.com

UERJ

Rua dos Ipês 678

27525-063 - Resende - RJ

Marina Maia Nogueira - marinamaianogueira@gmail.com

UERJ

Rua Geraldo Patrício 97

12244-647 - São José dos Campos - SP

Resumo: No estudo teórico de máquinas de fluxo motoras, destacam-se os diversos parâmetros de dimensionamento de ventiladores, que podem ser melhor compreendidos quando os alunos têm condições de interagir com o tema fisicamente, e desenvolver a percepção quanto aos efeitos secundários de um projeto bem sucedido. Este trabalho apresenta o desenvolvimento de uma bancada didática de ventiladores e os benefícios que a mesma contribui para o Curso de Engenharia Mecânica da UERJ, campus de Resende. Este desenvolvimento foi motivado pela ausência de experimentos no Laboratório de Motores, Hidráulica e Pneumática, para complementar o ensino teórico nas disciplinas de Fenômenos de Transporte e Máquinas de Fluxo. A bancada didática foi construída a partir de componentes já existentes no campus, de forma a permitir a aplicação dos conceitos estudados em livro texto do curso e que serão mostrados comparativamente neste material. Concluído este desenvolvimento, será possível aprimorar detalhes da técnica de projetos em ventiladores e desenvolver meios de análises teórico e experimental. Como resultado, além dos ganhos acadêmicos, os alunos terão maiores condições de estudo e 


\section{COBENCE - Evento onine

aperfeiçoamento tecnológico.

Palavras-chave: Bancada de ventiladores, Tubo de Pitot, Máquinas de fluxo 


\section{(C.COBENCE \\ DESENVOLVIMENTO DE UMA BANCADA DIDÁTICA PARA ANÁLISE COMPARATIVA DO DESEMPENHO DE VENTILADORES INDUSTRIAIS}

\section{INTRODUÇÃO}

O leque de aplicação dos diversos modelos de ventiladores na indústria é significativo e demanda por atenção frente as exigências de eficiência energética, custos operacionais e de processos, e por fim questões ambientais. Em muitas situações de projeto, o profissional é condicionado a administrar a tomada de decisão na especificação em produtos pré-definidos em catálogos dos fabricantes, onde na maioria das vezes estas decisões acabam por escolher equipamentos superdimensionados e/ou pouco adequados a aplicação de destino, gerando custos desnecessários.

O desenvolvimento de uma base de testes experimental, está em motivar os alunos do curso de engenharia em conciliar simulações numéricas aos modelos reais presentes nesta bancada, no complemento das atividades teóricas das disciplinas de Fenômenos de Transporte e Máquinas de Fluxo. Instigar o aluno ao ensino experimental, como apontado pela revista Nova Escola (2011), em que considera que existem duas condições para que a aprendizagem ocorra: o conteúdo deve ser potencialmente revelador e o estudante precisa se relacionar de maneira consistente, empírica e não arbitrária ao material trabalhado. Seguindo esta linha, a bancada foi construída a partir de equipamentos aproveitados da própria unidade acadêmica, possibilitando ao aluno desenvolver diversas atividades, a destacar:

- Mapeamento das pressões estáticas, dinâmica e total em diversos pontos ao longo do duto, utilizando tubos de Pitot e manômetros em "U" e inclinados;

- Análises das alturas manométricas, potências e rotação do ventilador para condições impostas ao sistema;

- Analise do sistema de ventilação e correlacionar aos parâmetros geométricos dos rotores e periféricos do conjunto;

- Aplicação das Leis da similaridade, através da variação de parâmetros como rotação, massa específica e pressão;

- Análise dos efeitos da perda de carga na tubulação e o perfil da camada limite. ventiladores:

A bancada experimental, foi desenvolvida com os seguintes modelos de

- Um ventilador axial, com um motor de $3 \mathrm{cv}$;

- Um ventilador centrífugo de acionamento indireto, pilotado por um motor de $1 \mathrm{cv}$;

- Um ventilador siroco pequeno (detalhe em amarelo), com um motor de $1 \mathrm{cv}$;

- Um ventilador siroco grande (detalhe em laranja), como visto na figura 1, pilotado por um motor de $3 \mathrm{cv}$.

Dentro da proposta acadêmica de ensino comparativo teórico e experimental, todos os ventiladores serão utilizados dentro de cada especificidade, para questões mais aprofundadas. Porém, inicialmente tomou-se como preferência o desenvolvimento didático no ventilador siroco pequeno (detalhe em amarelo) em função dos custos operacionais envolvidos e características físicas que somente neste modelo poderão ser empregadas, e no decorrer das discussões serão esclarecidas. 
Figura 1 - Bancada de Ventilador desenvolvida.

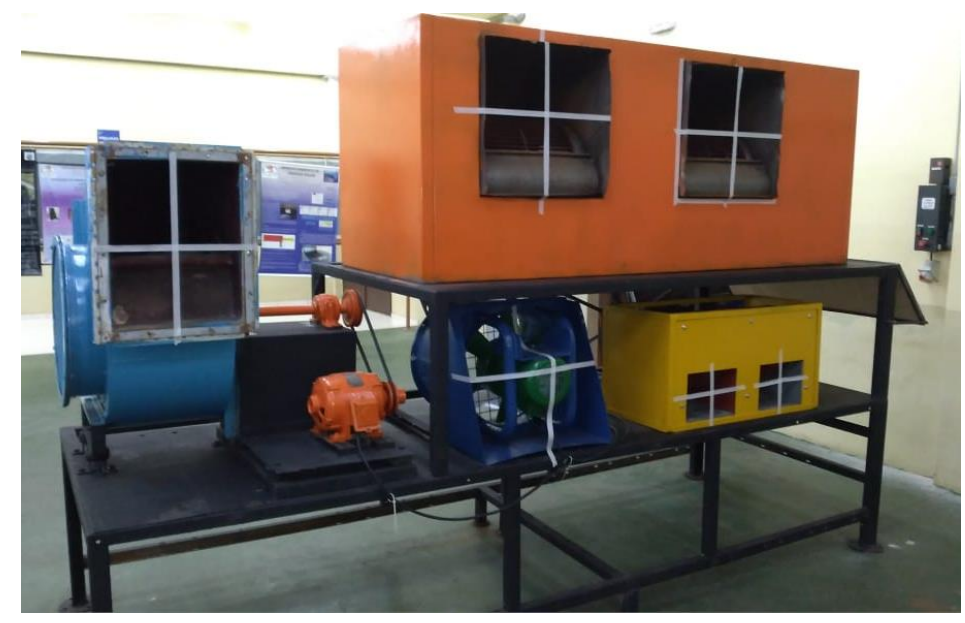

Fonte: O próprio autor

Esta bancada foi construída seguindo padrões de segurança através de um balanceamento da linha elétrica de alimentação, da utilização de conectores elétricos modelo Steck e de um sistema de acionamento elétrico por meio de um inversor de frequência modelo Siemens. Foram definidos protocolos de segurança para mapeamento e leitura de dados para garantir a proteção dos alunos, possibilitando o aprendizado e técnicas de seguras. O aluno estará em contato direto com o painel elétrico da bancada e com o inversor de frequência, fazendo com que ele tenha conhecimento de todo o processo de partida do motor do ventilador e de como o inversor de frequência é importante na mudança dos escoamentos e nos perfis de velocidade.

\section{METODOLOGIA}

O desenvolvimento de atividades práticas junto ao corpo discente, vão muito além da questão de mapear pontos, dados e ou resultados. Os alunos são estimulados através do questionamento experimental, de maneira sutil e desafiadora a desenvolver suas competências. O estímulo ao desenvolvimento das técnicas e a obtenção de resultados passam a ter um maior valor frente ao próprio experimento. As Diretrizes Curriculares Nacionais para os Cursos de Graduação em Engenharia (MEC, 2019), também comentado por (Gomes, 2019), apontam entre outras, as seguintes competências e habilidades gerais a serem estimuladas:

$\checkmark$ Aplicar conhecimentos matemáticos, científicos e tecnológicos e instrumentais a engenharia;

$\checkmark$ Projetar e conduzir experimentos e interpretar resultados;

$\checkmark$ Conceber, projetar e analisar sistemas, produtos e processos;

$\checkmark$ Identificar, formular e resolver problemas de engenharia;

$\checkmark$ Desenvolver e/ou utilizar novas ferramentas e técnicas;

$\checkmark$ Comunicar-se eficientemente nas formas escrita, oral e gráfica;

$\checkmark$ Atuar em equipes multidisciplinares;

$\checkmark$ Avaliar a viabilidade econômica de projetos de engenharia; 
Ao avaliar as competências acima, é possível identificar o quanto o propósito deste trabalho está alinhado nas inúmeras linhas de estudo e na formação do engenheiro, estimulando o desenvolvimento de habilidades através de metodologias ativas de ensino, onde o professor passa a ter um papel secundário no ensino, auxiliando os alunos na correta tomada de decisão, frente as linhas de propósito e raciocínio.

A bancada de ventiladores, tem inicialmente a proposta de complementar as disciplinas de Fenômenos de Transporte e Máquinas de Fluxo, porém posteriormente poderá ser explorada em outras disciplinas como Eletrotécnica, Instalações Industriais e Automação \& Controle. Nesta primeira etapa, as propostas serão:

$\Rightarrow$ Avaliar o perfil de similaridade do triângulo de velocidades entre os quatro (4) modelos de ventiladores, assim como medições de parâmetros de velocidade angular e vazão no rotor. Estimulando os alunos na análise do perfil aerodinâmico das hélices dos ventiladores e a desenvolver modelos analíticos comparativos aos dados reais, e gerar discussões das propriedades calculadas;

$\Rightarrow O$ ventilador siroco pequeno(detalhe em amarelo, figura 1 ), devido ao fato de apresentar uma maior regularidade em relação aos valores do perfil de velocidade do fluido em diversos pontos da seção transversal de saída, a proposta foi construir um túnel de vento neste equipamento (em detalhe na figura 3 ) e destacá-lo nas análises de elevação de pressão do sistema, avaliação das pressões (estática, dinâmica e total), análise da perda de carga, estudo da camada limite e em paralelo conciliar este equipamento com estudos de escoamento sobre perfis cilíndricos e ou placa plana para mapeamento térmico de temperaturas e trocas térmicas, trabalhados na disciplina de Fenômenos de Transporte. Sendo este ventilador pilotado através de um inversor de frequência, permitirá que os alunos se desenvolvam em diversas questões sob regime de escoamento laminar e turbulento.

A construção do túnel de vento será feita em madeirite, e geometricamente alinhado a norma americana de ventiladores AMCA 210, 2007 (Air Movement and Control Association), o duto deve ter seu comprimento equivalente a 10 vezes o seu diâmetro, conforme apresentado na figura 2. Para complementar as análises, será inserido ao final do duto uma tampa cônica (damper), conforme figura 3, para auxiliar na variação do fluxo de ar e proporcionar o estudo da elevação de pressão do sistema utilizando tubos de Pitot e manômetros inclinado, que serão construídos pelos próprios alunos. 
Figura 2 - Túnel de vento segundo a AMCA

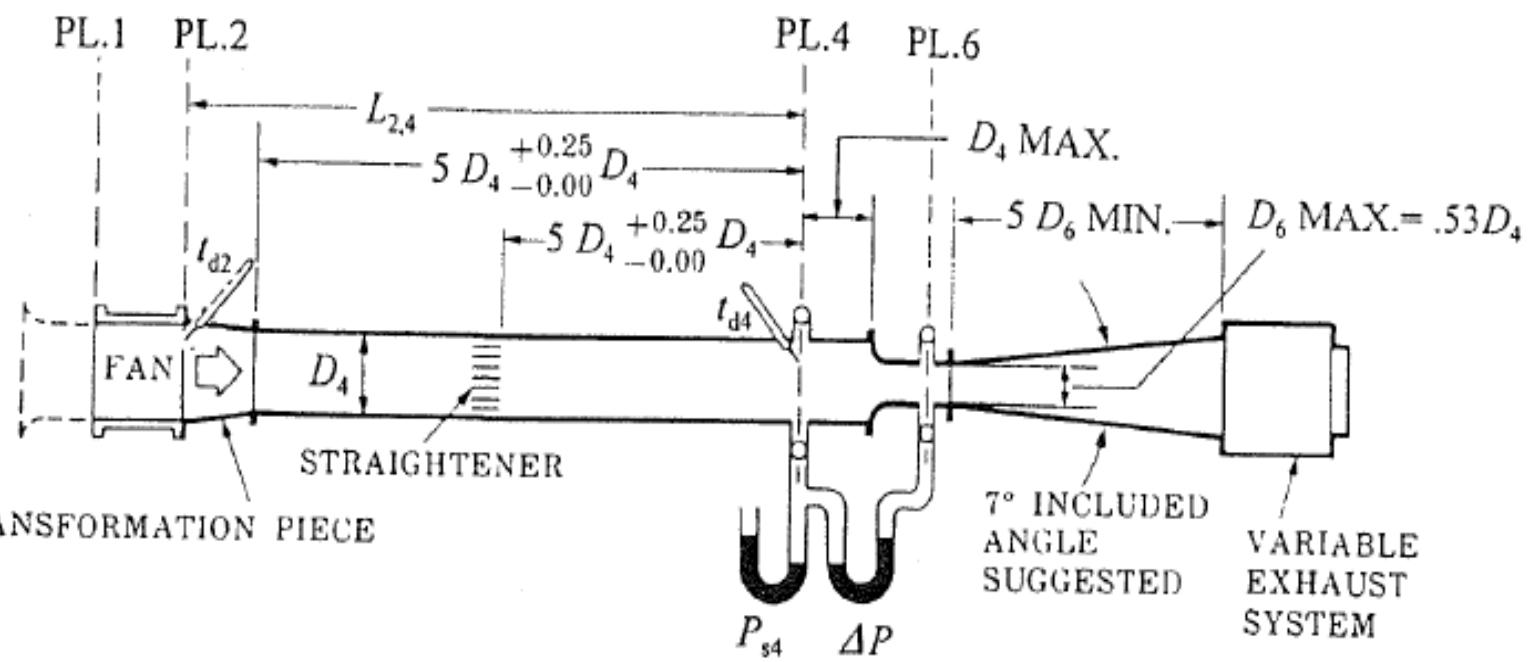

Fonte: AMCA 210: Laboratory Methods of Testing, 2007

Figura 3 - Túnel de vento do experimento UERJ.

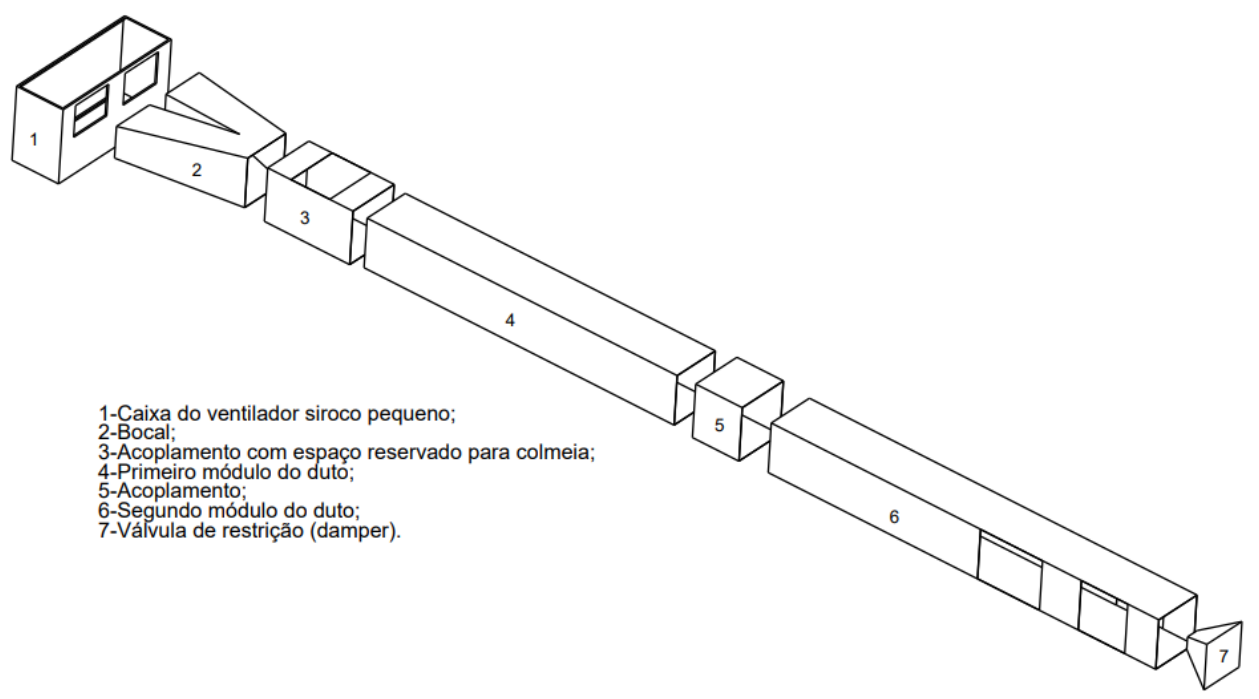

Fonte: O próprio autor

Como pode ser visto na figura 3 , o segundo módulo do duto possui duas partes vazadas, onde 0 primeiro recorte serve para visualização dos experimentos de escoamento sobre superfícies e estudos de trocas térmicas. Já o segundo recorte, serve como exaustão e monitoramento do ajuste que é feito pela válvula de restrição (damper), visto que ela possuirá uma escala graduada que possibilitará medir os efeitos internos de aumento de pressão dentro do duto de acordo com o avanço dessa válvula.

\subsection{Proposta para aplicação da metodologia}

O objetivo principal do projeto é inserir os alunos na análise experimental de ventiladores, estimular o conhecimento ao dimensionamento e projeto, conciliando os ensinamentos teóricos das disciplinas de máquinas de fluxo e fenômenos de transporte 
para que sejam implementados no decorrer do processo de análise experimental. Instigados pelo professor, que nesse momento atua como um moderador nas discussões, auxiliando os alunos na fixação dos conceitos através das orientações e nas formulações técnicas, em resposta aos resultados encontrados. A forma utilizada para aumentar essa interação é através da metodologia ativa, ou seja, o aluno é responsável pelas tomadas de decisões dentro do experimento, desde o momento em que se iniciam as análises até o fechamento das atividades. Estimulados a discussão, os resultados são abertamente analisados pelo grupo, no qual a contribuição de cada participante é valorizada, e contribui para a elevação de maturidade do grupo.

As primeiras análises propostas para os alunos a serem desenvolvidas, estão relacionadas aos 4 (quatro) modelos de ventiladores desta bancada, devido as diferenças conceituais destes equipamentos, os alunos são estimulados a avaliar as questões geométricas do perfil do rotor em cada caso e em seguida desenvolverem os respectivos triângulos de velocidades. Nesta análise, o aluno poderá realizar as medições físicas dos ângulos de inclinação das pás dos rotores, as medições das velocidades angulares e as vazões dos rotores. Com base nos dados reais, os alunos são incentivados a desenvolver as análises comparativas através dos modelos analíticos ajustados para cada modelo específico de ventilador.

Devido a questões financeiras, somente em um modelo de ventilador foi adequado a proposta de um túnel de vento. O modelo escolhido foi o ventilador siroco (foto1 detalhe em amarelo), este ventilador foi escolhido por apresentar melhores condições de vazão e velocidade uniforme, motor elétrico de baixa potência, excelentes resultados de custo benefício e segurança elétrica no manuseio junto ao corpo discente. O propósito deste experimento é estender as análises do próprio equipamento e, também, conciliar os estudos do escoamento e mapeamento da camada limite, estimular os alunos nas leituras de pressões de sucção e recalque, através de técnicas de utilização de manômetros em "U" e outros arranjos físicos declarados no item anterior deste artigo.

Ao começar a análise, os alunos são orientados a medir a temperatura e a pressão do ar utilizando um termômetro digital e um barômetro de mercúrio para definir as condições ambientais as quais os experimentos irão ocorrer. Com esses valores, é possível determinar a massa específica do ar $(\rho)$. Utilizando o inversor de frequência, a rotação do ventilador será fixada para controlar a variação de vazão do sistema de ventilação por meio da válvula de estrangulamento (damper). A área de saída do duto do ventilador será ajustada, desde $100 \%$ aberta até $0 \%$ fechada, e deste modo será possível obter as medidas das pressões estática, dinâmica e total, e por fim a velocidade do ar medido através do uso dos tubos de Pitot e de manômetros inclinados, e deste modo medir a vazão $(Q)$ do escoamento.

A pressão de estagnação $\mathrm{p}_{0}$ (ou pressão total) será obtida quando o fluido do escoamento for desacelerado até a velocidade zero através de um processo sem atrito. Para um escoamento incompressível, é utilizado a equação de Bernoulli para relacionar variações na velocidade e na pressão ao longo de uma linha de corrente, desprezando as diferenças de elevação (Fox e McDonald, 8ª edição).

$$
\frac{p}{\rho}+\frac{v^{2}}{2}=\text { constante }
$$

Considerando a pressão estática "p" em um ponto em que a velocidade é "V" e a pressão de estagnação " $\mathrm{p}_{0}$ " quando a velocidade for zero, temos: 


$$
p_{0}=p+\frac{\rho V^{2}}{2}
$$

Onde o termo $\frac{\rho V^{2}}{2}$ representa a pressão dinâmica do sistema. Ou seja, a pressão de estagnação ou pressão total, é o somatório das pressões estática e dinâmica. Através da pressão dinâmica é possível determinar a velocidade do escoamento em diversos pontos da seção transversal do duto.

A forma como o tubo de Pitot foi construído, permite que tanto o cálculo da vazão como da velocidade seja feito diretamente no painel de vidro fixado nos tubos em $U$ pelos alunos, possibilitando um trabalho de análise em conjunto e fazendo com que a imersão seja maior.

Figura 4 - Tubo de Pitot

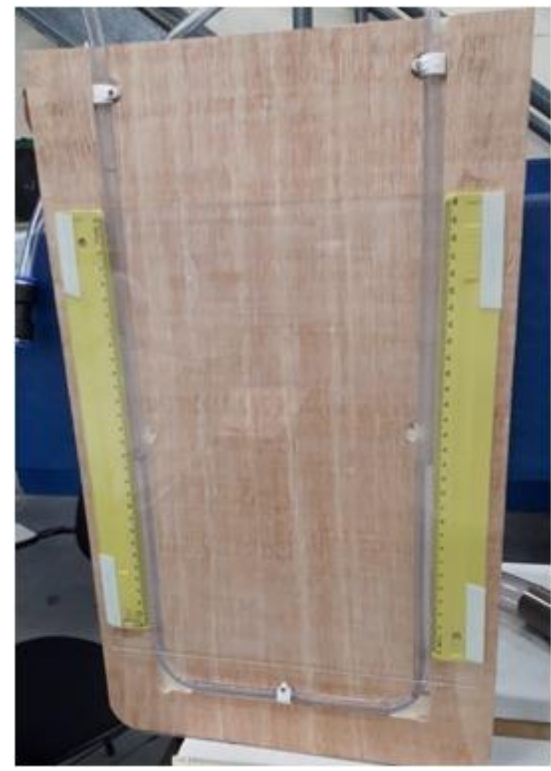

Fonte: O próprio autor (2021)

Com os valores encontrados de pressão total e vazão do ar, o aluno poderá gerar as curvas de funcionamento do ventilador por intermédio do uso das Leis de Similaridade das máquinas de fluxo, com isso, será possível traçar a curva característica do ventilador, o que torna possível a escolha do ventilador e a análise do comportamento frente a aplicação necessária. A curva característica de um ventilador é geralmente apresentada pelo seu fabricante para uma condição padrão, geralmente, $760 \mathrm{mmHg}, 20^{\circ} \mathrm{C}$ e $1,2 \mathrm{~kg} / \mathrm{m}^{3}$. No entanto, um ventilador somente irá operar nesta condição padrão em situação excepcional, pois a pressão atmosférica varia com a altitude do local e com as condições climáticas, a temperatura ambiente também será diferente e consequentemente a densidade de referência (Alé, 2011).

Devido a essas condições, a curva característica do ventilador deverá ser recalculada para uma condição média de operação utilizando o conceito de pressão total e as relações de similaridade de máquinas de fluxo. As três leis dos ventiladores são 
simplificações das equações mostradas a seguir junto com a equação da conservação da quantidade de movimento (Alé, 2011):

$$
\begin{aligned}
& Q_{2}=Q_{1}\left(\frac{n_{2}}{n_{1}}\right)\left(\frac{D_{2}}{D_{1}}\right)^{2} \\
& P_{2}=P_{1}\left(\frac{n_{2}}{n_{1}}\right)^{2}\left(\frac{D_{2}}{D_{1}}\right)^{2}\left(\frac{\rho_{2}}{\rho_{1}}\right) \\
& \dot{W}_{2}=\dot{W}_{1}\left(\frac{n_{2}}{n_{1}}\right)^{3}\left(\frac{D_{2}}{D_{1}}\right)^{5}\left(\frac{\rho_{2}}{\rho_{1}}\right)
\end{aligned}
$$

Onde:

$\mathrm{Q}$ - Vazão do $\operatorname{ar}\left(\mathrm{m}^{3} / \mathrm{s}\right)$

$\mathrm{P}$ - Pressão estática do ventilador $(\mathrm{Pa})$

$\dot{W}$ - Potência do ventilador (W)

$\mathrm{D}$ - Diâmetro do ventilador $(\mathrm{m})$

$\mathrm{n}$ - Rotação do rotor (RPM)

$\rho$ - Massa específica do fluido $\left(\mathrm{kg} / \mathrm{m}^{3}\right)$

Além dessa análise, é possível calcular o número de Reynolds e verificar o regime de escoamento do fluido neste túnel de vento. Para escoamento interno, o número de Reynolds é função do diâmetro hidráulico do duto, ou seja, é preciso calcular esse valor para determinar o regime do escoamento (Çengel, 2007).

$$
D_{h}=\frac{4 A}{2 p}
$$

Onde:

A - Área da seção transversal do duto $\left(\mathrm{m}^{2}\right)$

$2 p$ - Perímetro da seção transversal do duto $(m)$

Ao obter o diâmetro hidráulico da seção transversal, é possível calcular o número de Reynolds e analisar o valor para determinar o regime do escoamento do fluido (Çengel, 2007).

$$
\operatorname{Re}_{D}=\frac{\rho V D}{\mu}
$$

Onde: 
$v=\frac{\mu}{\rho}-$ Viscosidade cinemática $\left(\mathrm{m}^{2} / \mathrm{s}\right)$

$\mathrm{V}$ - Velocidade média do escoamento $(\mathrm{m} / \mathrm{s})$

D - Diâmetro hidráulico da seção transversal $(m)$

Cada regime de escoamento possui uma faixa do número de Reynolds o qual define o regime de escoamento do fluido, com o valor do número de Reynolds calculado é possível observar em qual faixa esse escoamento se encontra (Çengel, 2007).

\author{
$\operatorname{Re} \leq 2300$ - Escoamento laminar \\ $2300 \leq R e \leq 4000$ - Escoamento de transição \\ $\operatorname{Re} \geq 4000$ - Escoamento turbulento
}

\title{
3 RESULTADOS E DISCUSSÃO
}

Neste trabalho, foi apresentado a proposta do desenvolvimento de uma bancada de ventiladores e a adequação para atender as atividades experimentais de diversas disciplinas do curso de Engenharia Mecânica, da UERJ-Resende. O projeto atualmente está em fase de construção, e acumula um certo atraso na execução, devido a questão da pandemia do COVID-19. Porém, não é a primeira vez que trabalhos com características semelhantes a este são desenvolvidos nesta unidade, e compartilhados a comunidade acadêmica, através do COBENGE, como exemplos:

- Desenvolvimento de uma bancada hidráulica de alta pressão, no complemento de ensino para elaboração de projetos de engenharia (Cordeiro, L., 2019 - COBENGE);

- Construção de uma bancada didática para análise de performance veicular (Cordeiro, L., 2019 - COBENGE);

O desenvolvimento de atividades como estas, representam um legado de orgulho para os alunos egressos, através da contribuição na qualidade do ensino, e como reflexo ao grau de maturidade, responsabilidade e de conhecimento técnico adquirido por estes futuros profissionais. Por outro lado, a Universidade estimulando estas ações, possibilita uma melhor redistribuição de orçamentos financeiros, beneficiando atender a diversos níveis acadêmicos dentro da instituição.

O objetivo de trabalhar o conceito no dimensionamento de máquinas de fluxo através de arranjos experimentais foi assertivo, visto que o grau de desenvolvimento técnico dos alunos foi melhorado devido a possibilidade de desenvolver modelos analíticos com base em dados físicos e medições reais.

Através deste experimento, os alunos serão capazes de diferenciar os modelos de ventiladores existentes com base nos princípios físicos de funcionamento e aplicação, mapeando as principais características dos detalhes construtivos, assim como assimilar maiores informações sobre questões extra classe como especificações de motores elétricos, utilização de inversores de frequência e o manuseio de diversos equipamentos de medições e de segurança, aproximando mais o aluno da realidade profissional. 
A primeira análise sugerida no experimento, será o mapeamento da geometria do rotor de cada ventilador e traçar o triângulo de velocidades adequado a cada modelo conceitual do equipamento, possibilitando destacar as diferenças e características de cada um.

Já o túnel de vento proporciona uma experiência única na vida acadêmica dos alunos, pois é através deste equipamento que empresas aeronáuticas, automobilísticas e de outros segmentos de mercado conseguem realizar testes em seus produtos. Então através deste experimento, os alunos conseguem entender o funcionamento de um túnel de vento e suas características, observar a variação de pressão através do tubo de Pitot, realizar cálculos relacionados a máquinas de fluxo, observar a troca térmica de um corpo aquecido sendo resfriado por meio da convecção forçada e, ainda, observar os tipos de escoamento e a transição entre eles.

O desenvolvimento desta bancada está propiciando aos autores o desenvolvimento do trabalho de conclusão de curso de graduação em Engenharia Mecânica na UERJ e possibilitando diversas análises e estudos, garantindo um desenvolvimento pessoal e profissional.

\section{CONSIDERAÇÕES FINAIS}

Através deste projeto, é possível entender a importância da contribuição técnica na formação dos alunos em complemento as disciplinas de Fenômenos de Transporte e em Máquinas de Fluxo, ao longo do curso de graduação. Além dos experimentos proporcionarem uma boa didática, a metodologia ativa auxilia ampliar a visão dos alunos para questões de Engenharia Mecânica e no crescimento pessoal e profissional. Esse tipo de abordagem, em que o aluno é a chave principal para o desenvolvimento das atividades, é fundamental na formação de engenheiros, na melhoria das habilidades, na resolução de problemas reais, permitindo a quebra de barreira que existe entre a teoria e as situações que ocorrem de fato dentro de uma análise real.

Outro ponto a ser observado nesta bancada é a diversidade de estudos e pesquisas que ela proporciona além dos que estão sendo feitos neste artigo. Como sugestão para trabalhos futuros, ainda é possível realizar um estudo mais aprofundado sobre camada limite, assim como conciliar a utilização de fumaça ou gelo seco dentro do duto mostrando o comportamento do escoamento em torno de um objeto. Por fim, a construção de um freio de Prony no mancal do eixo do motor do ventilador siroco, possibilita o registro do torque do motor elétrico. Conhecendo esse valor, os alunos podem calcular a potência mecânica $\left(P_{m}\right)$ gasta pelo motor acoplado ao ventilador e por meio da altura de elevação $(H)$ determinar a potência hidráulica $\left(P_{\text {hid }}\right)$ do ventilador $e$, assim, calcular o seu rendimento ( $\eta$ ).

\section{REFERÊNCIAS BIBLIOGRÁFICAS}

AIR MOVEMENT AND CONTROL ASSOCIATION. AMCA 210: informação e documentação. Arlington Heights, IL, 2007.

ALÉ, Jorge Villar. Sistemas de Ventilação Industrial: Ventiladores. 2011. Apostila Departamento de Engenharia Mecânica e Mecatrônica. Pontifícia Universidade Católica do Rio Grande do Sul, Porto Alegre, 2011. Disponível em: 
https://issuu.com/jorgeantoniovillarale/docs/apostila ventiladores/10. Acesso em: 09 abr. 2021.

ÇENGEL, Yunus A.; CIMBALA, John M. Mecânica dos Fluidos Fundamentos e Aplicações. 1. ed, Editora AMGH. 2007.

FERNANDES, Elisângela. David Ausubel e a Aprendizagem Significativa. Nova Escola, 01 dez. 2011. Disponível em: https://novaescola.org.br/conteudo/262/davidausubel-e-a-aprendizagem-significativa. Acesso em: 01 abr. 2021.

FOX, Robert W.; PRITCHARD, Philip J.; MCDONALD, Alan T. Introdução à Mecânica dos Fluidos. 8. ed, Editora LTC. 2014.

GOMES, Francisco de Salles Cintra. A extensão universitária e a promoção de competências na formação do aluno de engenharia. In: XLVII Congresso Brasileiro de Educação em Engenharia e II Simpósio Internacional de Educação em Engenharia da ABENGE, 2019, Fortaleza. Anais. Pontifícia Universidade Católica de Campinas Faculdade de Engenharia Elétrica. Disponível em: http://www.abenge.org.br/cobenge/2019/anais.php. Acesso em: 01 abr. 2021.

MINISTÉRIO DA EDUCAÇÃO - CONSELHO NACIONAL DE EDUCAÇÃO. Diretrizes Curriculares Nacionais do Curso de Graduação em Engenharia. Brasília, 23 jan. 2019.

Disponível

em:

http://portal.mec.gov.br/index.php?option=com docman\&view=download\&alias=109871pces001-19-1\&category slug=marco-2019-pdf\&Itemid=30192. Acesso em: 01 abr. 2021.

PEREIRA, Renzo Caldellas. Ensaio de Desempenho de Ventiladores em uma Bancada Didática. 2014. Monografia (Graduação) - Curso de Engenharia Mecânica. Universidade Federal do Espírito Santo, Vitória, 2014. Disponível em: https://mecanica.ufes.br/sites/engenhariamecanica.ufes.br/files/field/anexo/2014-

2 renzo caldellas pereira.pdf. Acesso em: 06 abr. 2021.

SANTIAGO, Rodrigo Silveira. Análise Numérica e Experimental de um Ventilador Radial. 2018. Monografia (Graduação) - Curso de Engenharia Automotiva. Universidade Federal de Santa Catarina, Joinville, 2018. Disponível em: https://repositorio.ufsc.br/handle/123456789/191861. Acesso em: 12 abr. 2021. 


\title{
DEVELOPMENT OF A DIDACTIC BENCH FOR COMPARATIVE COMPARATIVE ANALYSIS OF INDUSTRIAL FAN PERFORMANCE
}

\begin{abstract}
In the theoretical study of flow machines, the different fan sizing parameters stand out, which can be better understood when students can interact with the topic physically, and develop the perception of the effects of a successful project. This work presents the development of a didactic bench for fans and the benefits that it contributes to the Mechanical Engineering Course at UERJ, Resende campus. This development was motivated by the absence of experiments in the Hydraulics, and Pneumatics Laboratory, to complement the theoretical teaching in the disciplines of Transport Phenomena and Flow Machines. The didactic bench was built from components already existing on campus, to allow the application of the concepts studied in the course's textbook and which will be shown comparatively in this material. Once this development is completed, it will be possible to improve details of the fan design technique and develop means of theoretical and experimental analysis. As a result, in addition to academic gains, students will have greater conditions for study and technological improvement.
\end{abstract}

Key-words: Fan bench, Pitot tube, Flow machines. 